\title{
Dupla ampliação anterior e posterior do anel aórtico para substituição valvar
}

\author{
João Ricardo SANT'ANNA *, Renato A. K. KALIL *, Paulo R. PRATES *, \\ Carlos JUNGBLUT **, Ivo A. NESRALLA *
}

Sant'Anna J R, Kalil R A K, Prates P R, Jungblut C, Nesralla I A - Dupla ampliação anterior e posterior do anel aórtico para substituição valvar. Rev Bras Cir Cardiovasc 2002; 17 (1): 35-46.

RESUMO: Objetivo: Apresentar técnica de dupla ampliação anterior e posterior do anel aórtico, que difere das previamente descritas por utilizar enxertos distintos, para implante de prótese valvar e avaliar os resultados obtidos em 26 pacientes.

Método: O procedimento é realizado com circulação extracorpórea convencional. Para substituição de valva nativa ou prótese aórtica estenótica, ampliação anelar posterior decorre da incisão no ponto médio do seio não coronário, que é estendida ao folheto mitral anterior; ampliação anterior resulta de incisão no anel aórtico anterior, estendida por $2 \mathrm{~cm}$ no septo interventricular, não transfixado. Reconstrução aórtica (e ampliação anelar) é realizada mediante enxertos de pericárdio bovino distintos, sendo um triangular para incisão anterior e outro elíptico para incisão posterior. Após a fixação da prótese (mecânica ou biológica), a aorta ascendente é suturada com auxílio destes enxertos. Defeitos cardíacos associados são corrigidos.

Resultados: A operação aumentou o diâmetro do anel aórtico de $18,0 \pm 2,6 \mathrm{mmHg}$ para 24,5 $\pm 2,1 \mathrm{mmHg}(\mathrm{p}<0,01)$. Na operação, um paciente $(3,8 \%)$ requereu revascularização miocárdica por obstrução dos óstios coronários. No período pós-operatório imediato um paciente foi reoperado por sangramento e outro faleceu por choque séptico (morbidade e mortalidade de 3,8\%). Durante seguimento médio de 24 meses não ocorreram óbitos ou reoperações, sendo observada melhora na classe funcional em 23 pacientes acompanhados por mais de 3 meses. Nos pacientes com estenose da valva ou prótese aórtica, o gradiente sistólico máximo ao ecocardiograma Doppler mostrou uma redução de $87,3 \pm 20,8 \mathrm{mmHg}$, valor pré-operatório, para $25,9 \pm 10,3 \mathrm{mmHg}(\mathrm{p}<0,01)$.

Conclusão: A ampliação anterior e posterior mediante enxertos distintos resultou em aumento importante do anel aórtico e, estando associada com baixa mortalidade e morbidade, pode ser considerada nas obstruções severas da via de saída do ventrículo esquerdo ou para reconstrução anelar aórtica.

DESCRITORES: Valva aórtica, cirurgia. Estenose da valva aórtica, cirurgia. Implante de prótese de valva.

\footnotetext{
Trabalho realizado no Instituto de Cardiologia do Rio Grande do Sul. Fundação Universitária de Cardiologia. Porto Alegre, RS, Brasil. * Da Equipe de Cirurgia Cardiovascular do Instituto de Cardiologia do Rio Grande do Sul.

** Estagiário Bolsista da FABIC.

Endereço para correspondência: João R. Sant'Anna. Instituto de Cardiologia do Rio Grande do Sul. Unidade de Pesquisa. Av. Princesa Isabel, 395. Porto Alegre, RS, Brasil. CEP: 90620-001. Telefax: (51) 230-3600 R3777. e-mail: pesquisa@cardnet.tche.br
} 
Sant'Anna J R, Kalil R A K, Prates P R, Jungblut C, Nesralla I A - Dupla ampliação anterior e posterior do anel aórtico para substituição valvar. Rev Bras Cir Cardiovasc 2002; 17 (1): 35-46.

\section{INTRODUÇÃO}

Embora se reconheça que as próteses aórticas projetadas mais recentemente ofereçam baixos gradientes transvalvares, por vezes é necessário utilizar uma técnica de ampliação do anel aórtico para implantar um dispositivo protético de maior diâmetro. Isto pode ocorrer quando se observa que o diâmetro do anel valvar nativo é muito pequeno, por malformação congênita, constituição anatômica ou presença de prótese reconhecidamente estenótica. Também pacientes pediátricos podem necessitar de ampliação anelar aórtica, na pressuposição de que o crescimento corporal não será acompanhado pelo aumento do diâmetro protético e determinará um aumento progressivo do gradiente transprotético.

Visando reduzir a possibilidade de manutenção da obstrução na via de saída do ventrículo esquerdo durante a substituição valvar aórtica (ou o aparecimento tardio desta obstrução), diversas técnicas de ampliação anelar foram desenvolvidas (1-4). Mostram diferentes graus de complexidade, determinam aumento variável no diâmetro anelar aórtico e não são desprovidas de risco. Uma abordagem cirúrgica que complemente as opções existentes para o cirurgião quando este necessite ampliar o anel aórtico é cabível e, se adequada, poderá se tornar útil adjunto cirúrgico.

O presente estudo visa propor uma técnica de ampliação anterior e posterior do anel aórtico para a operação de substituição valvar e avaliar os resultados iniciais obtidos em um grupo de pacientes submetidos ao procedimento.

\section{CASUÍSTICA E MÉTODOS}

\section{Pacientes}

De janeiro de 1993 a agosto de 1999, foram operados no Instituto de Cardiologia do Rio Grande do Sul / Fundação Universitária de Cardiologia 26 pacientes para operação de dupla ampliação anterior e posterior do anel valvar e implante de prótese aórtica, isolada ou em associação com outros procedimentos intracardíacos. Destes pacientes, 9 eram de sexo feminino e 17 masculinos, com uma idade variando entre 4 e 43 anos (média de 20,6 $\pm 11,7$ anos). A Tabela 1 expressa características dos pacientes.

TABELA 1 CARACTERÍSTICAS DEMOGRÁFICAS DOS PACIENTES SUBMETIDOS A AMPLIAÇÃO SIMÉTRICA DO ANEL AÓRTICO

\begin{tabular}{|c|c|c|c|c|c|c|}
\hline $\mathrm{N}$ & SEXO & $\begin{array}{l}\text { IDADE } \\
\text { (anos) }\end{array}$ & ETIOLOGIA & $\begin{array}{l}\text { PROCEDIMENTO PRÉVIO } \\
\text { (última intervenção) }\end{array}$ & $\begin{array}{l}\text { tempo } \\
\text { (anos) }\end{array}$ & $\begin{array}{c}\text { C.F. } \\
\text { (NYHA) }\end{array}$ \\
\hline 1 & feminino & 12 & congênita & $\begin{array}{l}\text { ressecção anel sub-Ao + } \\
\text { plastia Ao (Trusler) }\end{array}$ & 8 & IV \\
\hline 2 & masculino & 4 & congênita & $\begin{array}{l}\text { Ventriculosseptoplastia + } \\
\text { plastia Ao (Trusler) }\end{array}$ & 1 & II \\
\hline 3 & masculino & 21 & reumática & prótese Ao + plastia M & 8 & II \\
\hline 4 & masculino & 23 & reumática & prótese aórtica & 1 & II \\
\hline 5 & feminino & 12 & congênita & plastia Ao com balão & 2,5 & III \\
\hline 6 & masculino & 24 & congênita & prótese Ao (ampliação) & 11 & II \\
\hline 7 & feminino & 30 & congênita & prótese Ao (ampliação) & 11 & II \\
\hline 8 & masculino & 26 & reumática & prótese Ao & 12 & II \\
\hline 9 & masculino & 11 & reumática & plastia Ao (Trusler) & 3 & II \\
\hline 10 & masculino & 33 & congênita & prótese Ao & 8 & II \\
\hline 11 & feminino & 40 & reumática & prótese Ao + prótese $M$ & 4 & II \\
\hline 12 & masculino & 13 & reumática & prótese Ao & 3 & IV \\
\hline 13 & feminino & 40 & congênita & prótese Ao (ampliação) & 5 & III \\
\hline $14^{*}$ & feminino & 25 & reumática & prótese Ao + prótese M & 9 & II \\
\hline 15 & masculino & 12 & congênita & plastia Ao com balão & 4 & II \\
\hline 16 & masculino & 13 & congênita & plastia Ao com balão & 2 & II \\
\hline 17 & masculino & 15 & congênita & prótese aórtica & 15 & II \\
\hline 18 & masculino & 10 & congênita & plastia Ao com balão & 4 & ||̂ \\
\hline 19 & masculino & 12 & congênita & prótese Ao & 7 & II \\
\hline 20 & masculino & 14 & reumática & prótese Ao + prótese $M$ & 5 & II \\
\hline 21 & masculino & 19 & congênita & prótese Ao & 2 & II \\
\hline 22 & masculino & 9 & congênita & plastia Ao com balão & 1 & II \\
\hline 23 & feminino & 5 & congênita & plastia Ao com balão & 4 & III \\
\hline 24 & feminino & 43 & reumática & prótese Ao + prótese $M$ & 7 & III \\
\hline 25 & masculino & 17 & congênita & plastia Ao com balão & 1 & II \\
\hline 26 & feminino & 42 & reumática & prótese Ao + prótese M & 2 & II \\
\hline$x^{-1}$ & & 20,8 & & & & \\
\hline E.P & & 11,7 & & & & \\
\hline
\end{tabular}

NOTA : Paciente $14^{*}$ faleceu no pós-operatório imediato.

ABREVIATURAS: C.F. (NYHA): Classe funcional conforme New York Heart Association Ao: aórtica ; M : mitral X: média; E.P.: erro padrão. 
Sant'Anna J R, Kalil R A K, Prates P R, Jungblut C, Nesralla I A - Dupla ampliação anterior e posterior do anel aórtico para substituição valvar. Rev Bras Cir Cardiovasc 2002; 17 (1): 35-46.

A lesão valvar aórtica original era estenose em 22 e insuficiência em 4 pacientes. Todos os pacientes haviam sido submetidos previamente a algum tipo de intervenção na valva aórtica nativa, sendo os procedimentos assim definidos: valvoplastia aórtica por balão ${ }^{(5)}$ em $7(26,9$ $\%)$ pacientes e pelo menos uma operação cardíaca prévia com circulação extracorpórea em 19 (73,1 \%). Destes pacientes, 3 submeteramse à plastia valvar aórtica (6) isolada ou associada a ressecção de anel subvalvular aórtico ou ventriculosseptoplastia; 10 submeteram-se a substituição valvar aórtica isolada e nos demais 6 foi associado à substituição valvar aórtica um procedimento na valva mitral (plastia valvar em 1 e substituição protética em 5). Quanto ao número de intervenções prévias, 4 pacientes haviam sido submetidos a duas operações cardíacas, 3 a três e 1 a quatro procedimentos, com um total de $8(30,8 \%)$ pacientes submetidos a múltiplas operações prévias.

Dos pacientes submetidos ao implante de prótese aórtica, em 3 havia sido realizada ampliação posterior do anel aórtico com enxerto de Dacron ou de pericárdio bovino preservado em glutaraldeído, conforme técnica descrita por Nicks (1).

O diagnóstico que motivou a indicação cirúrgica de implante de prótese aórtica e ampliação anelar foi a estenose da valva nativa em 8 pacientes (associada a obstrução subvalvar em um e a pseudoaneurisma da aorta ascendente em outro), insuficiência valvar aórtica em 2 (associada a comunicação interventricular residual em um) e estenose de prótese aórtica em 16.

\section{Técnica Cirúrgica e Cuidados Per-Operatórios}

Os pacientes realizaram avaliação pré-operatória de rotina $(7)$.

A operação compreendeu técnicas bem estabelecidas. Esternotomia mediana foi utilizada para abordar o coração. As bolsas na aorta e no átrio direito foram confeccionadas com fio de poliéster trançado 00 e o coração foi canulado. Circulação extracorpórea foi conduzida com hipotermia sistêmica moderada entre 28 e $32^{\circ} \mathrm{C}$, mediante oxigenador de bolhas descartável (primeiros pacientes da série) ou de membrana capilar. O coração foi protegido por infusão de solução cardioplégica hipotérmica cristalóide (solução de St. Thomas II resfriada a $4{ }^{\circ} \mathrm{C}$ ), injetada diretamente nos óstios coronarianos com cânulas apropriadas.
Após incisão aórtica longitudinal e infusão de solução cardioplégica, a valva ou prótese aórtica foi inspecionada e removida. A aortotomia foi estendida inferior e posteriormente até o anel valvar aórtico, pela porção média do seio de Valsalva correspondente ao folheto não coronariano, e prolongada através do anel e folheto mitral anterior (1). Nos pacientes portadores de prótese mitral, a incisão posterior ficou limitada ao anel aórtico. Nas reoperações foi efetuada uma pequena dissecção externa a aorta, junto à conexão da veia cava superior com o átrio direito e sobre o teto do átrio esquerdo, visando liberação da parede aórtica e maior dilatação do anel valvar.

A ampliação anelar aórtica anterior decorreu de incisão na parede aórtica relacionada à artéria pulmonar, iniciada no ponto médio da aortotomia existente e seguindo pela comissura aórtica entre os folhetos coronarianos direito e esquerdo. $\mathrm{O}$ anel aórtico foi secionando e a incisão estendida por $2 \mathrm{~cm}$ no septo interventricular, a uma profundidade máxima de $0,5 \mathrm{~cm}$. Quando a comissura anterior não foi localizada devido à remoção da prótese previamente implantada, o ponto médio entre os óstios coronarianos foi utilizado para indicar o local da secção anelar anterior. Foi ainda realizada uma dissecção limitada do anel valvar, externamente a parede aórtica adjacente à incisão. A Figura $1 \mathrm{~A}$ indica a disposição do campo cirúrgico após realização das incisões de ampliação.

A reconstrução da via de saída do ventrículo esquerdo foi iniciada na incisão aórtica anterior, pelo implante de um enxerto de pericárdio bovino preservado em glutaraldeído. Este enxerto, de forma triangular para permitir a ampliação pretendida, foi suturado de modo contínuo a partir da extremidade inferior da incisão septal com fio de polipropileno monofilamentar 4-0. A sutura seguiu pelos bordos da incisão ventricular, anel aórtico e parede aórtica, sendo interrompida na linha da aortotomia longitudinal.

Após, foi efetuada a reconstrução do anel aórtico posterior, também mediante enxerto de pericárdio bovino preservado em glutaraldeído. Este enxerto foi confeccionado com forma elíptica alongada, para permitir a reconstrução de toda a aortotomia longitudinal ao término da sutura. A sutura contínua com fio de polipropileno monofilamentar 3-0 ou 4-0 foi iniciada ao nível da valva mitral e estendeu-se pelo anel aórtico e parede aórtica, aonde foi temporariamente interrompida. Ao término da dupla ampliação anterior e posterior, o diâmetro do anel aórtico foi reavaliado, visando verificar o tamanho da prótese 
Sant'Anna J R, Kalil R A K, Prates P R, Jungblut C, Nesralla I A - Dupla ampliação anterior e posterior do anel aórtico para substituição valvar. Rev Bras Cir Cardiovasc 2002; 17 (1): 35-46.

a ser implantada. A Figura 1B mostra os enxertos posicionados, previamente ao implante da prótese aórtica.

A prótese aórtica foi implantada através de pontos em fio de poliéster trançado 2-0 ancorados em almofadas de feltro de Dacron, que foram passados no anel aórtico nativo e em sua projeção nos enxertos de pericárdio bovino. Nestes enxertos, as suturas foram efetuadas do exterior para o interior da aorta, cuidando-se para que fosse mantido o plano de assentamento para a prótese. Quando possível, a musculatura superficial do infundíbulo do ventrículo direito foi utilizada para auxiliar a fixação dos pontos correspondentes ao enxerto de pericárdio bovino anterior, visando reduzir o risco de sangramento pela incisão no septo interventricular. Os pontos de fixação da válvula foram reparados individualmente e, concluída a sutura no anel aórtico, passados através do anel da prótese, conforme Figura 2A. A prótese foi posicionada e fixada e a aortorrafia concluída pela sutura do enxerto posterior. A Figura 2B mostra a prótese aórtica em posição, previamente a sutura de reconstrução da parede aórtica com os enxertos de ampliação.

Conjuntamente com a ampliação do anel aórtico e implante da prótese aórtica, em 5 pacientes foram realizados procedimentos reparadores adicionais no coração (reconstrução da aorta ascendente em 1, ventriculosseptoplastia em 1 e implante de prótese mitral em 3). Na correção de defeitos intracardíacos, estes procedimentos foram efetuados após preparo do anel aórtico para ampliação, mas antecedendo a sutura de enxertos e implante da prótese valvar aórtica.

Realizados os procedimentos intracardíacos, a operação foi concluída de modo rotineiro e os pacientes encaminhados à Sala de Recuperação PósOperatória, onde receberam cuidados previamente descritos (8). Após 48 horas, os pacientes foram transferidos para a Unidade de Cuidados Pós-Opera-

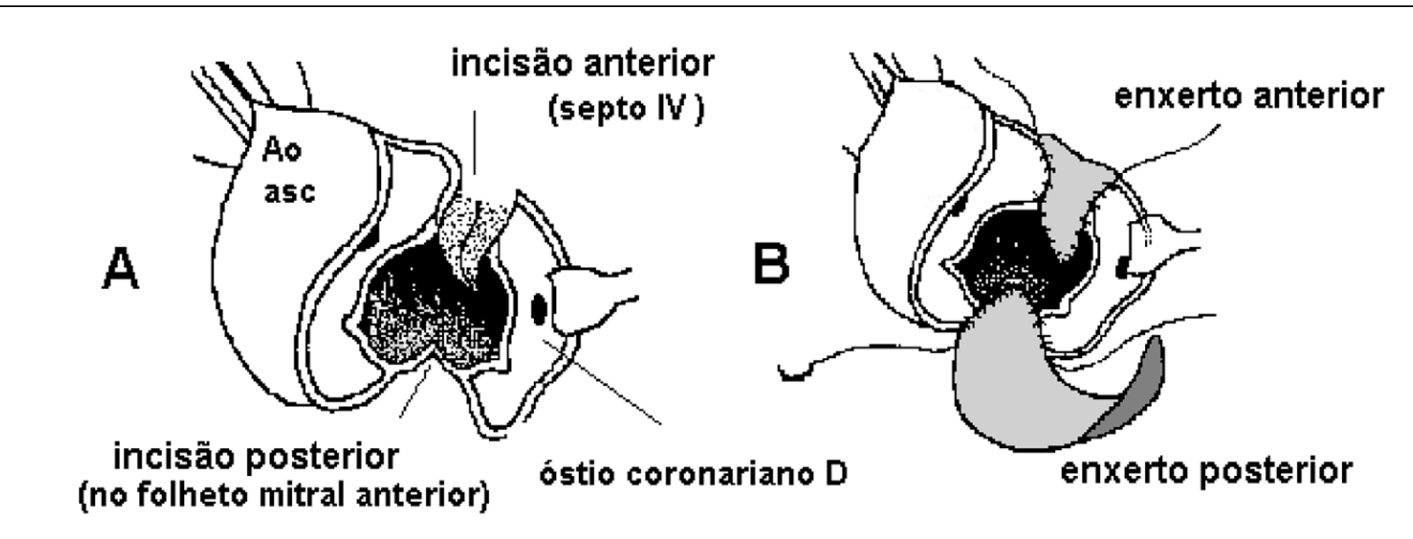

Fig. 1 - Cirurgia de ampliação simétrica. (A) Incisões posterior (contínua a linha da aortotomia) e anterior (compreendendo o septo interventricular). (B) Sutura dos enxertos de ampliação. Abreviaturas: Ao Asc: aorta ascendente; septo IV: septo interventricular; D: direito.

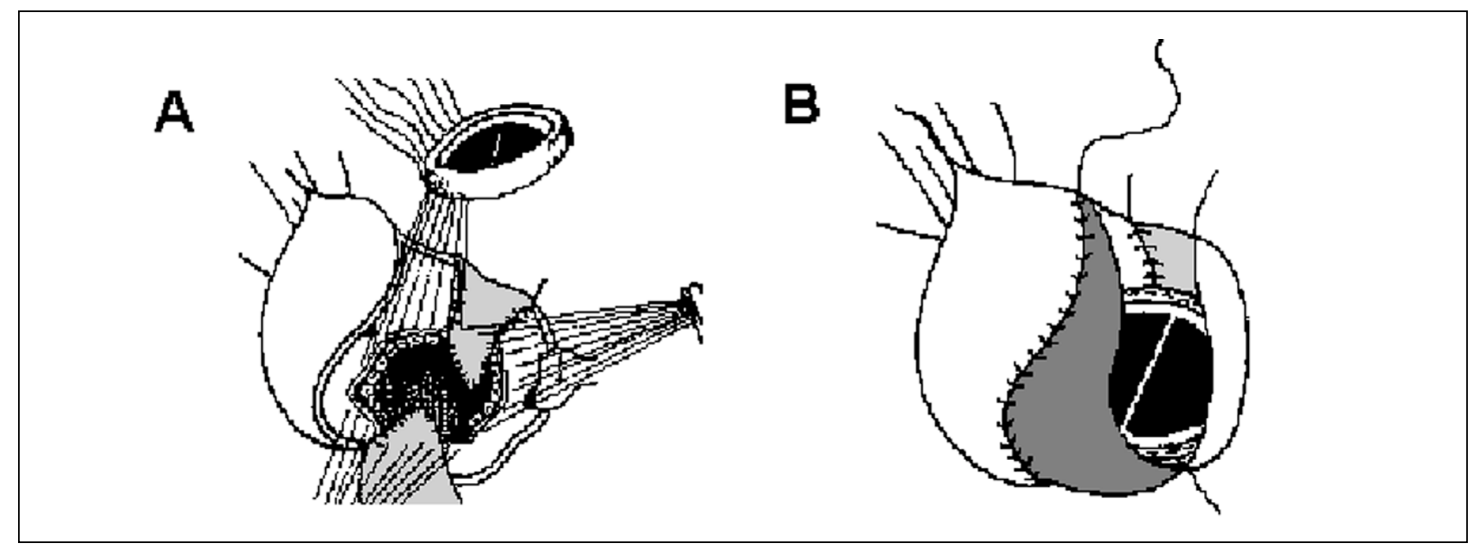

Fig. 2 - Conclusão da operação de ampliação: A. Pontos de fixação da prótese aórtica. B . Reconstrução da parede aórtica com os enxertos de pericárdio bovino utilizados na ampliação anelar. 
Sant'Anna J R, Kalil R A K, Prates P R, Jungblut C, Nesralla I A - Dupla ampliação anterior e posterior do anel aórtico para substituição valvar. Rev Bras Cir Cardiovasc 2002; 17 (1): 35-46.

tórios II, aonde permaneceram internados até a alta hospitalar. Por ocasião da alta hospitalar, os pacientes foram encaminhados ao clínico assistente ou ao ambulatório da Instituição. Para os pacientes que receberam próteses valvares mecânicas foi mantida anticoagulação oral com femprocumona em dosagem adequada para manter o tempo de protrombina entre 20 e $30 \%$ do valor controle, o que foi aferido mensalmente.

\section{Avaliação da Operação}

A técnica cirúrgica proposta foi estudada quanto ao aumento no diâmetro do anel aórtico obtido pelo procedimento, a mortalidade e morbidade decorrentes, a qualidade de vida dos pacientes sobreviventes e a repercussão sobre o coração, este avaliado mediante ecocardiografia bidimensional com Doppler (9). Um ecocardiograma foi realizado em todos os pacientes antes da operação e objetivou-se pelo menos uma avaliação em variável tempo de pós-operatório; a avaliação foi dirigida para a via de saída do ventrículo esquerdo na região subvalvar aórtica, a presença de regurgitação protética ou para-protética e a determinação do gradiente sistólico instantâneo máximo entre ventrículo esquerdo e aorta.

Para coleta de informações, foi pesquisado o prontuário hospitalar, revisto o arquivo informatizado do Setor de Cirurgia e realizada consulta médica com os pacientes. As informações coletadas foram colocadas em tabelas de contin- gência e submetidas a tratamento estatístico pelo teste "t" de Student para dados pareados. Os dados foram expressos como valor absoluto, valor médio ou valor percentual \pm erro padrão. O nível de significância estipulado foi um alfa crítico de $5 \%$, sendo que valores de $p$ inferiores a 0,05 foram referidos quando identificados.

\section{RESULTADOS}

\section{Avaliação Intra-Operatória}

A operação para dupla ampliação anterior e posterior do anel aórtico propiciou aumento do diâmetro médio do anel valvar de $18,0 \pm 2,6 \mathrm{~mm}$ para $24,5 \pm 2,1 \mathrm{~mm}(p<0,01)$. Se considerarmos pacientes com valva aórtica nativa, estes valores variaram de $17,0 \pm 3,0 \mathrm{~mm}$ para $23,4 \pm 2,1$ $\mathrm{mm}(\mathrm{p}<0,01)$ como mostra o Gráfico 1. Para os pacientes em que a prótese aórtica foi substituída, a ampliação resultou em aumento de $19,0 \pm 2,2 \mathrm{~mm}$ para $25,1 \pm 1,8 \mathrm{~mm}(\mathrm{p}<0,01)$, como ilustra o Gráfico 2. A Tabela 2 indica a variação individual do anel valvar, bem como o tamanho e modelo de próteses implantadas.

Para realização das correções cirúrgicas foi necessário um tempo de circulação extracorpórea variável entre 88 e 265 minutos, com média de $117,0 \pm 36,2$ minutos. O período de pinçamento aórtico foi de 70 a 123 minutos, com média de 86,0 $\pm 19,1$ minutos, conforme Tabela 2 .

\section{GRÁFICO 1}

VARIAÇÃO NO DIÂMETRO DO ANEL AÓRTICO EM 10 PACIENTES COM VALVA NATIVA $(17,0+1,2 \mathrm{~mm}$ para $23,4+2,1 \mathrm{~mm} ; \mathrm{p}<0,01)$

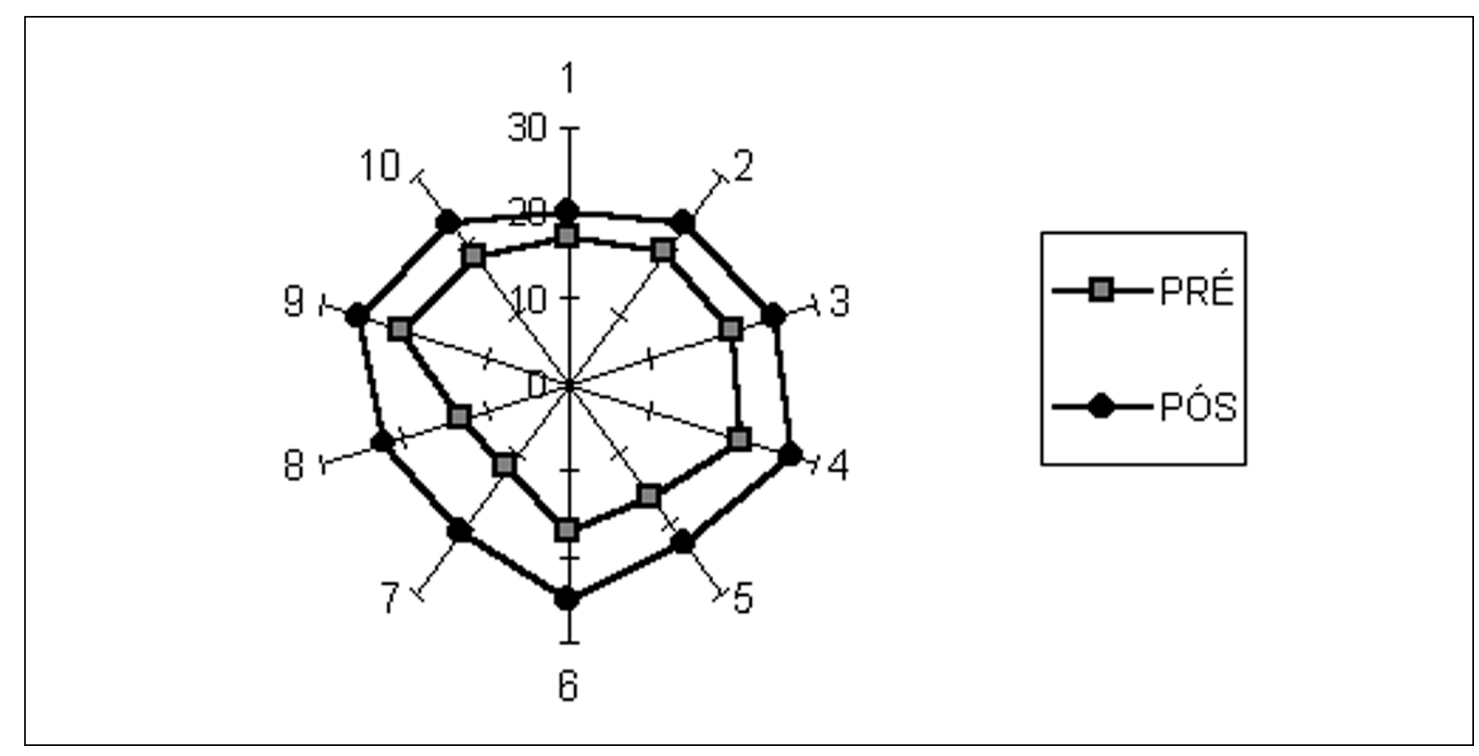


Sant'Anna J R, Kalil R A K, Prates P R, Jungblut C, Nesralla I A - Dupla ampliação anterior e posterior do anel aórtico para substituição valvar. Rev Bras Cir Cardiovasc 2002; 17 (1): 35-46.

GRÁFICO 2

VARIAÇÃO NO DIÂMETRO DO ANEL AÓRTICO EM 16 PACIENTES COM PRÓTESE ESTENÓTICA $(19,0+2,2 \mathrm{~mm}$ para $25,1+1,8 \mathrm{~mm} ; \mathrm{p}<0,01)$
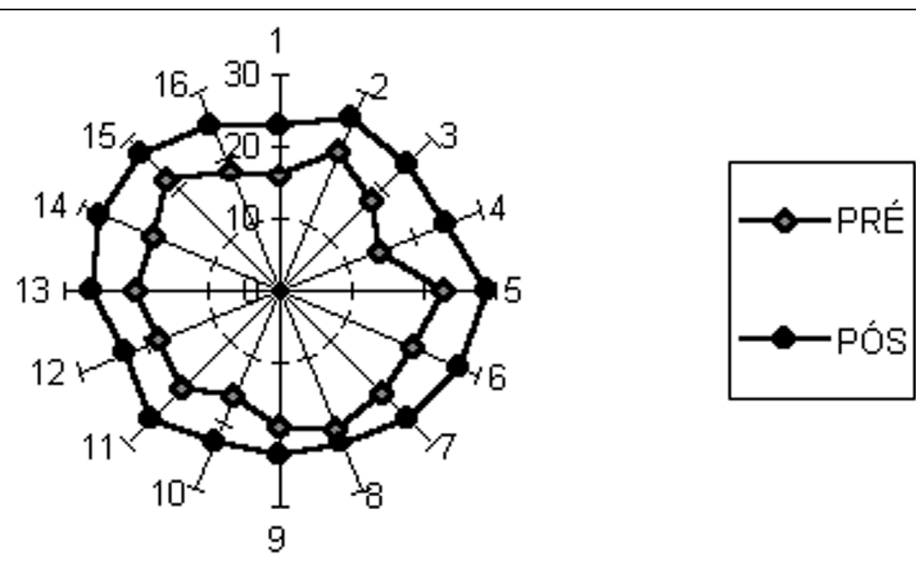

TABELA 2

\begin{tabular}{|c|c|c|c|c|c|c|c|}
\hline \multicolumn{8}{|c|}{ AUMENTO DO DIÂMETRO DO ANEL AÓRTICO E PROCEDIMENTOS CIRÚRGICOS REALIZADOS } \\
\hline \multirow[t]{2}{*}{$\mathbf{n}$} & \multicolumn{2}{|c|}{$\begin{array}{l}\text { diâmetro } \\
\text { do anel }\end{array}$} & \multicolumn{2}{|c|}{$\begin{array}{l}\text { modelo e tamanho de } \\
\text { prótese valvar }\end{array}$} & \multirow{2}{*}{$\begin{array}{l}\text { procedimento } \\
\text { cirúrgico } \\
\text { associado }\end{array}$} & \multicolumn{2}{|c|}{$\begin{array}{c}\text { duração de } \\
\text { procedimento }\end{array}$} \\
\hline & pré & pós & explantada & implantada & & CEC & Isquemia \\
\hline 1 & 17 & 20 & valva nativa (E.B.) & Sorin 19 (Björk) & $\begin{array}{l}\text { ressecção de anel sub Ao } \\
\text { reconstrução da Ao asc. }\end{array}$ & 122 & 86 \\
\hline 2 & 19 & 23 & valva nativa & Sorin 21 (Björk) & ventriculosseptoplastia & 116 & 103 \\
\hline 3 & 16 & 23 & Omniscience 14 & CarboMedics 21 & Carbomedics M27 & 144 & 120 \\
\hline 4 & 21 & 26 & Sorin 21 (Björk) & Carbomedics 23 & & 100 & 81 \\
\hline 5 & 20 & 25 & valva nativa & bioprótese 23 & & 88 & 55 \\
\hline 6 & 18 & 25 & Lilehey-Kaster 16 & CarboMedics 23 & & 100 & 80 \\
\hline 7 & 15 & 25 & Lilehey-Kaster 14 & Carbomedics 23 & & 97 & 80 \\
\hline 8 & 23 & 29 & Starr-Edwards 11 & Medtronic 27 & & 106 & 91 \\
\hline 9 & 21 & 27 & valva nativa & Carbomedics 25 & & 97 & 71 \\
\hline 10 & 20 & 27 & Björk-Shiley 19 & Carbomedics 25 & & 121 & 97 \\
\hline 11 & 20 & 25 & Omniscience 19 & Carbomedics 23 & & 126 & 80 \\
\hline 12 & 21 & 23 & bioprótese Ao (E.B.) & Carbomedics 21 & reconstrução anel Ao & 137 & 105 \\
\hline 13 & 19 & 23 & bioprótese Ao 21 & Carbomedics 21 & reconstrução anel Ao & 90 & 70 \\
\hline 14 * & 18 & 25 & Lilehey-Kaster 16 & Omniscience 23 & limpeza da prótese M & 118 & 103 \\
\hline 15 & 16 & 23 & valva nativa & St. Jude 21 & & 103 & 89 \\
\hline 16 & 17 & 25 & valva nativa & St. Jude 23 & & 90 & 76 \\
\hline 17 & 16 & 23 & Lilehey-Kaster 16 & St. Jude 23 & 2 pontes de safena & 265 & 123 \\
\hline 18 & 12 & 21 & valva nativa & St. Jude 21 & & 91 & 74 \\
\hline 19 & 19 & 25 & Lilehey-Kaster 19 & St. Jude 23 & & 130 & 86 \\
\hline 20 & 18 & 23 & Sorin 21 & St. Jude 21 & St. Jude M27 & 158 & 128 \\
\hline 21 & 20 & 26 & Omniscience 21 & Sorin 23 (moderna) & reconstrução anel Ao & 92 & 74 \\
\hline 22 & 13 & 22 & valva nativa & St. Jude 21 & & 135 & 103 \\
\hline 23 & 20 & 25 & valva nativa & bioprótese 23 & & 96 & 82 \\
\hline 24 & 19 & 27 & Omniscience 19 & St. Jude 25 & St. Jude M27 & 157 & 125 \\
\hline 25 & 18 & 23 & valva nativa & St. Jude 23 & & 136 & 85 \\
\hline 26 & 22 & 27 & St. Jude 21 & St.Jude 25 & limpeza da prótese $\mathrm{M}$ & 139 & 113 \\
\hline$x$ & 18,0 & $24,5^{\star *}$ & & & & 117,0 & 86,0 \\
\hline EP & 2,6 & 2,1 & & & & 36,2 & 19,1 \\
\hline
\end{tabular}

NOTA : diâmetro do anel aórtico expresso em $\mathrm{mm}$, conforme avaliado no campo operatório.

Paciente $14^{*}$ faleceu no pós-operatório imediato.

ABREVIATURAS: C.E.C.: circulação extracorpórea; ISQUEMIA: tempo de pinçamento aórtico; Ao : aórtico (a); asc. : ascendente; $M$ : mitral; EB : endocardite bacteriana; $X$ : valor médio; EP : erro padrão.

NÍVEL DE SIGNIFICÂNCIA : ** : variação significativa para $\mathrm{P}<0,01$. 
Sant'Anna J R, Kalil R A K, Prates P R, Jungblut C, Nesralla I A - Dupla ampliação anterior e posterior do anel aórtico para substituição valvar. Rev Bras Cir Cardiovasc 2002; 17 (1): 35-46.

\section{Morbidade e Mortalidade Hospitalar}

Não ocorreu nenhum óbito durante a operação.

Um paciente submetido a implante isolado de bioprótese aórtica de pericárdio bovino 23 apresentou hemorragia moderada no local da ampliação anterior, após o término da circulação extracorpórea. Pela localização do sangramento, inferiu-se como originário da incisão do septo interventricular e o tratamento consistiu em sutura do bordo do ventrículo direito sobre o enxerto de pericárdio de reconstituição da parede aórtica anterior, o que determinou hemostasia na área.

A maior dificuldade transoperatória ocorreu em um paciente com óstios coronários próximos ao anel da prótese previamente implantada; após remoção desta, o segmento de aorta compreendido entre os óstios coronários e o anel valvar ficou reduzido, porém aparentemente adequado para o implante da nova prótese. Contudo, ocorreu obstrução das artérias coronárias e foi necessário o implante de 3 pontes de safena aortocoronária (para coronárias direita, descendente anterior e ramo marginal da artéria circunflexa) visando corrigir a complicação.

Ocorreu um óbito no $4^{\circ}$ dia após a operação, devido a choque séptico e falência de múltiplos órgãos, perfazendo mortalidade de 3,8 \%.

Como complicação imediata, ocorreu drenagem mediastinal aumentada implicando na revisão cirúrgica em um paciente (3,8 \%). Não foi encontrado sangramento nas suturas relativas a ampliação aórtica ou em qualquer outro local do coração, mas foram observados alguns pontos hemorrágicos na parede posterior do esterno. A evolução do paciente foi satisfatória após a reoperação.

Receberam alta hospitalar 25 (96,2\%) pacientes. Durante o acompanhamento, não se obteve informações de um paciente $(3,8 \%$ do total) e 24 foram acompanhados por período variável entre 3 e 60 meses, com média de $24,0 \pm 20,1$ meses. Neste período não foram registrados óbitos ou complicações relacionadas ao procedimento.

Na última avaliação clínica de 24 pacientes, $22(84,6 \%)$ estavam assintomáticos (classe fun- cional I, critérios da NYHA), não mostravam sinais de insuficiência cardíaca. Dois pacientes $(7,7 \%)$ mostram sintomas de cansaço e dispnéia aos grandes esforços (classe funcional II, NYHA). O Gráfico 3 indica a situação atualizada de todos os pacientes.

\section{GRÁFICO 3 \\ SITUAÇÃO DOS PACIENTES QUANDO DA ÚLTIMA EVOLUÇÃO}

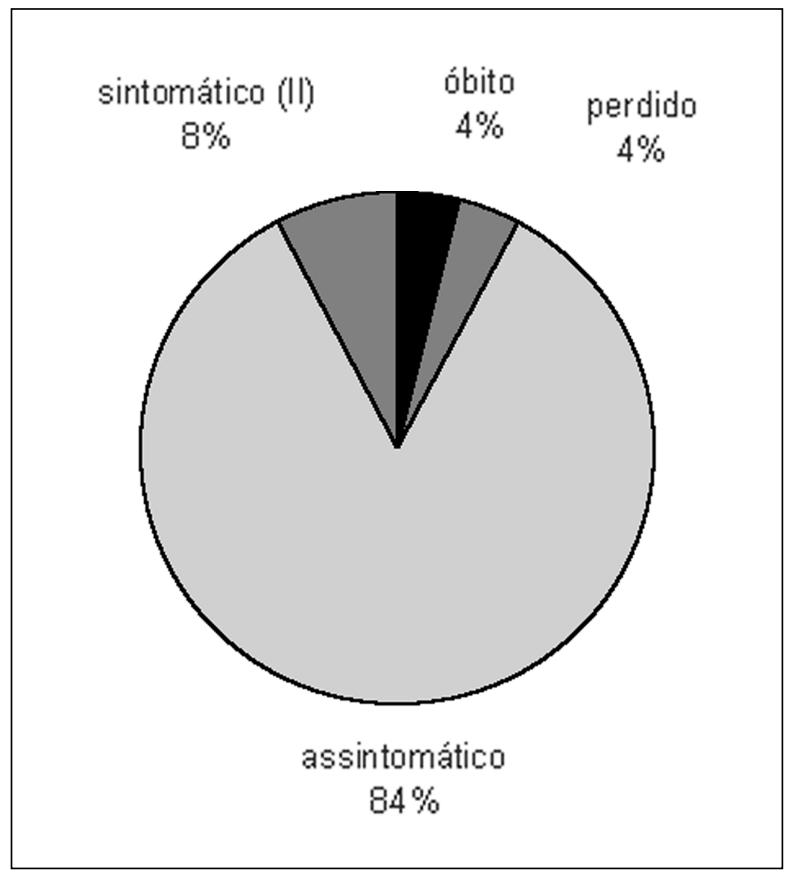

\section{Acompanhamento Ecocardiográfico}

Foi observada uma significativa redução do gradiente sistólico instantâneo máximo da via de saída do ventrículo esquerdo para os pacientes com estenose valvar ou protética: de um valor médio pré-operatório de $87,3 \pm 20,8 \mathrm{mmHg}$ houve diminuição para 25,9 $\pm 10,3 \mathrm{mmHg}$ ( $\mathrm{p}<$ $0,01)$. Para 5 pacientes com valva aórtica nativa estenótica, o gradiente sistólico reduziu de 92,4 $\pm 21,7 \mathrm{mmHg}$ para 27,0 $\pm 9,1 \mathrm{mmHg}(\mathrm{p}<0,01)$. Em 11 pacientes com prótese estenótica (excluídos 1 paciente falecido, 1 perdido e $3 \mathrm{com}$ alta hospitalar mais recente e sem ecocardiograma controle pós-operatório tardio), o gradiente sistólico máximo reduziu de $85,0 \pm 21,7 \mathrm{mmHg}$ para $25,5 \pm 11,2 \mathrm{mmHg}(p<0,01)$. 
Os Gráficos 4 e 5 indicam a variação individual do gradiente sistólico máximo.

A observação da via de saída do ventrículo esquerdo, realizada nos estudos ecocardiográficos pós-operatórios, não mostrou estruturas obstrutivas relacionadas com a sutura subprotética aórtica dos enxertos de pericárdio bovino utilizados nas ampliações anterior e posterior.

\section{GRÁFICO 4}

REDUÇÃO NO GVSVE $(\mathrm{mmHg})$ EM 5 PACIENTES COM VALVA AÓRTICA NATIVA ESTENÓTICA $(92,4+21,7 \mathrm{mmHg}$ para $27,0+9,1 \mathrm{mmHg} ; \mathrm{p}<0,01)$

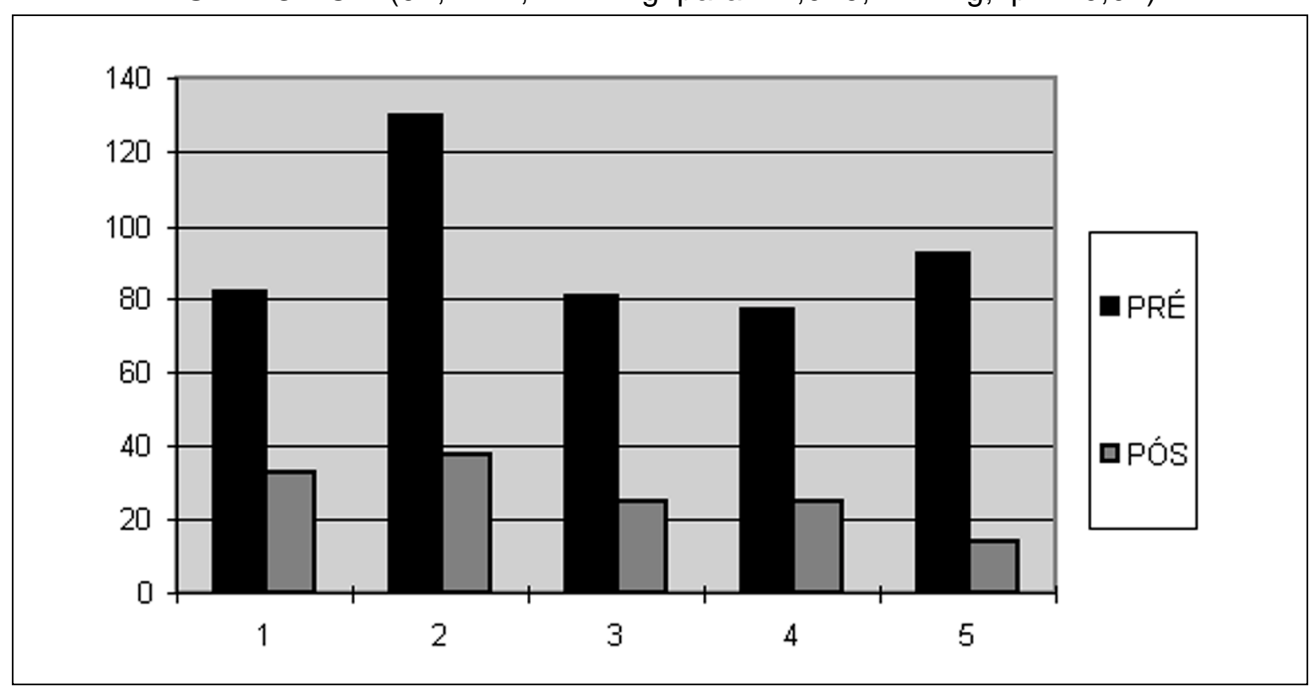

\section{GRÁFICO 5}

REDUÇÃO NO GVSVE $(\mathrm{mmHg})$ EM 11 PACIENTES COM PRÓTESE AÓRTICA

ESTENÓTICA $(85,0+21,7 \mathrm{mmHg}$ para $25,5+11,2 \mathrm{mmHg} ; \mathrm{p}<0,01)$

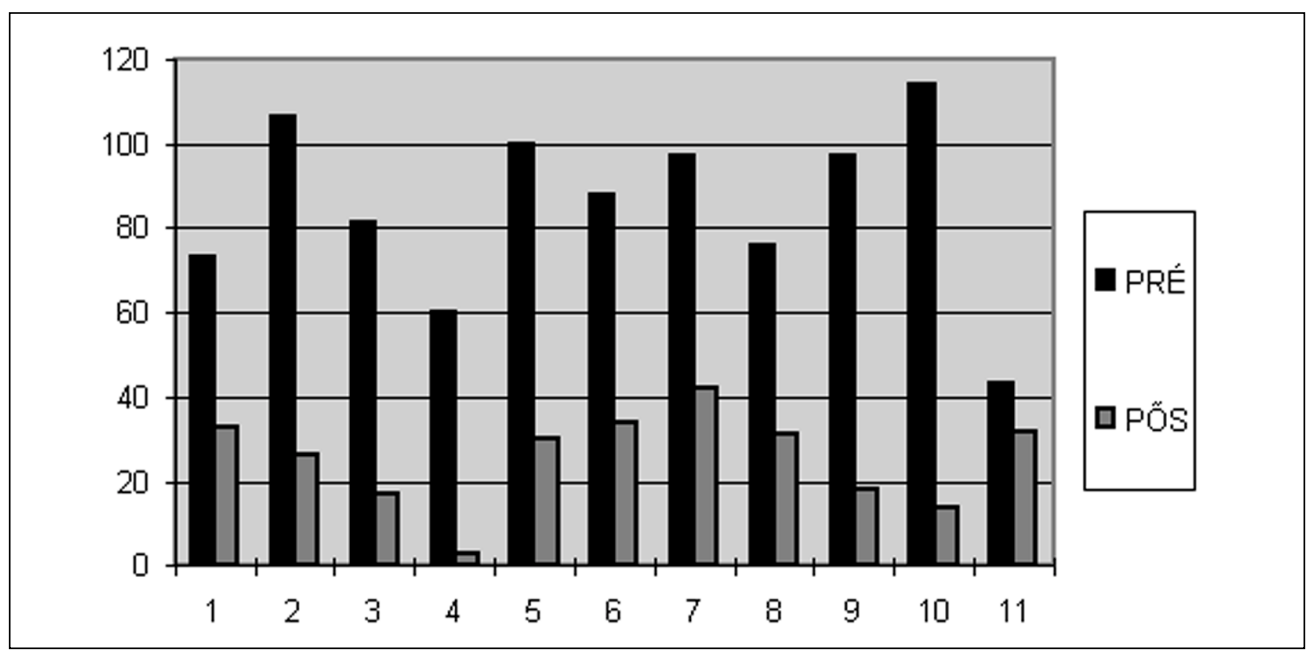

\section{COMENTÁRIOS}

Na presença de um orifício aórtico de diâmetro reduzido com relação ao valor normal, o cirurgião deve lançar mão de uma técnica de substituição valvar que permita o implante de um substituto de maior diâmetro do que 0 do anel valvar. Algumas alternativas técnicas possibilitam um ganho de um tamanho da prótese, o que pode ser suficiente.
A primeira opção é o implante de uma prótese de desempenho hemodinâmico satisfatório nos diâmetros reduzidos, como os modelos St. Jude e CarboMedics $(10,11)$. Uma alternativa é o implante da prótese aórtica acima do anel valvar $(12,13)$, quando foi utilizada prótese mecânica com ângulo de abertura do disco de 70 graus, inferior ao de outras próteses como o modelo Medtronic Hall, que tem abertura de 75 graus. Nos modelos de maior aber- 
Sant'Anna J R, Kalil R A K, Prates P R, Jungblut C, Nesralla I A - Dupla ampliação anterior e posterior do anel aórtico para substituição valvar. Rev Bras Cir Cardiovasc 2002; 17 (1): 35-46.

tura, o posicionamento inclinado pode determinar uma abertura do disco além do eixo do fluxo sangüíneo, resultando no fechamento intermitente da prótese na diástole (14).

Outro recurso é a incisão limitada no septo interventricular (ou miomectomia) sob a comissura entre o folheto coronário direito e o esquerdo. Ela permite uma maior distensão dos tecidos adjacentes ao anel e o implante de uma prótese um número maior do que a normalmente compatível com anel aórtico presente. Esta ampliação limitada da via de saída do ventrículo esquerdo permite resultado similar ao apresentado pela substituição valvar usual (15).

As técnicas de ampliação do orifício aórtico são utilizadas quando um grande ganho no diâmetro do anel aórtico é necessário; tornam possível o implante de uma prótese aórtica maior em 2 ou mais tamanhos do que a permitida pelo anel nativo. Elas podem ser classificadas de acordo com o local anatômico aonde a transecção anelar é realizada.

A ampliação anelar posterior, mediante transecção do anel valvar e extensão da incisão até o folheto anterior da valva mitral, foi descrita com variações relacionadas ao local exato da incisão no anel aórtico e a forma/constituição do enxerto utilizado na reconstrução aórtica. Uma técnica consiste de aortotomia longitudinal estendida a parede lateral e posterior da aorta, secionando o anel aórtico na região média do folheto não coronariano $(1,16)$. A incisão aórtica é prolongada através do anel valvar mitral, por até $1 \mathrm{~cm}$ no folheto anterior. A reconstrução subanelar é realizada pela sutura de um enxerto elíptico. O aumento na circunferência do orifício anelar alcança de 1 a $2 \mathrm{~cm}$. Alternativa cirúrgica para ampliação posterior é a extensão da aortotomia através da comissura entre o folheto não coronário e o coronário esquerdo, até a junção do anel aórtico e mitral; neste caso, o folheto mitral não necessita ser incisado $(2,17)$. O átrio esquerdo é dissecado e afastado, se possível sem ser aberto, embora isto freqüentemente ocorra. Um amplo enxerto é suturado a partir da parte inferior da incisão, com um reforço externo com feltro, visando reduzir o sangramento.

Os resultados comunicados para a ampliação posterior variam em função de fatores como tipo de procedimento, época da operação (e é necessário recordar que progressos foram feitos deste a introdução das técnicas na década de 1970) e variação na população operada. A mortalidade operatória, inicialmente de $40 \%$ (1) ou $25 \%$ (2), reduziu-se a próximo de zero (18-20). Complicações relativas à função da valva mitral foram citadas, sendo mais grave a ruptura na sutura do enxerto no local de reconstrução do folheto anterior.

Quando da substituição das valvas mitral e aórtica, uma alternativa é o emprego do folheto mitral anterior para ampliar o anel aórtico. Este folheto é liberado das cordoalhas de sustentação e rebatido superiormente para dentro da aorta através do átrio esquerdo, possibilitando a ampliação do anel aórtico, a fixação posterior da prótese aórtica e a reconstrução da parede aórtica ${ }^{(21)}$. A substituição valvar mitral é realizada mediante atriotomia esquerda convencional.

A ampliação anterior do anel aórtico é realizada por técnica conhecida como aortoventriculoplastia ou operação de Konno-Rastan ${ }^{(1,2)}$. Esta técnica é destinada primariamente a patologias que associem a estenose valvar aórtica com uma obstrução a nível subvalvar, como o túnel aorto-ventricular; mas pode ser empregada na estenose valvar aórtica isolada, especialmente quando se deseja maior ampliação anelar, como em pacientes pediátricos ou mesmo adultos (22-24).

Por se tratar de procedimento extenso, a aortoventriculoplastia implica em elevado risco cirúrgico e se associa a complicações significativas em alguns pacientes. Embora a experiência recente dos grupos que realizam o procedimento tenha demonstrado uma significativa queda na mortalidade (que era de $50 \%$ nos comunicados originais), têm sido registradas complicações como o bloqueio intraventricular e átrio-ventricular ou o defeito residual no septo interventricular. A função ventricular esquerda e direita se mantém preservada apesar da incisão e da reconstrução do septo interventricular, que em alguns pacientes mostra movimento paradoxal (22). Em um número limitado de procedimentos, usualmente envolvendo reoperação, a combinação de uma ampliação posterior com a aortoventriculoplastia foi empregada para obtenção de adequado diâmetro de anel aórtico $(20,25)$.

Uma técnica para ampliação combinada posterior e anterior mediante uso de enxerto único foi descrita por YAMAGUCHI et al.(26). Contudo, a incisão anterior utilizada não é estendida através do anel aórtico.

A substituição da raiz aórtica por enxerto composto (prótese implantada em enxerto tubular), homoenxerto aórtico (27) ou autoenxerto pulmonar $(28,29)$ é um procedimento originalmente proposto para operação da dissecção da aorta associada a comprometimento valvar. Tem sido também empregado na endocardite valvar aórtica ou em deformidades complexas da valva e pode mesmo ser combinado com a aortoventricu-loplastia, visando tratar a estenose aórtica recorrente em túnel ou a estenose valvar aórtica associada a anel aórtico hipoplásico de crianças. Neste caso foi referido como substituição estendida da raiz aórtica $(27,30)$.

A revisão das técnicas disponíveis para ampliação anelar aórtica indica que alguns dos procedimentos compreendidos na operação proposta neste artigo foram utilizados previamente. A ampliação posterior do anel aórtico é bem conhecida $(1,2,17)$. A secção anterior do anel aórtico é realizada na 
Sant'Anna J R, Kalil R A K, Prates P R, Jungblut C, Nesralla I A - Dupla ampliação anterior e posterior do anel aórtico para substituição valvar. Rev Bras Cir Cardiovasc 2002; 17 (1): 35-46.

aortoventriculoplastia, embora sendo o local indicado o seio coronariano direito, entre a comissura do folheto coronariano direito e esquerdo e o óstio coronariano direito ${ }^{(3,4)}$. Uma incisão anterior compreendendo o anel pulmonar foi avaliada experimentalmente em cães ${ }^{(32)}$. A forma final das incisões na aorta constitui uma letra $Y$ invertida, lembrando a sugerida para ampliação da estenose supravalvar aórtica, com a finalidade de evitar distorções e corrigir disfunção da valva natural (33). Mais recentemente foi apresentada uma técnica denominada ampliação bilateral do anel aórtico, utilizando um enxerto único em forma de $Y^{(26)}$. As duas extremidades da bifurcação são empregadas na reconstrução da incisão anterior (que não aborda o septo interventricular) e da incisão aórtica posterior (que não aborda o folheto mitral anterior). Enxertos de diversos materiais vêm sendo empregados para suturar nichos e reconstituir o anel aórtico quando da operação por endocardite bacteriana (34). Enxerto de pericárdico bovino preservado em glutaraldeído tem sido amplamente utilizado no país para reparo de defeitos intracardíacos e constituição ou ampliação da parede aórtica.

O grau de originalidade da técnica proposta prende-se a abordagem anterior mediante secção do anel aórtico na comissura anterior, incisão longitudinal no septo interventricular muscular para ampliação da circunferência da via de saída do ventrículo esquerdo e anel aórtico e na reconstrução da continuidade entre a parede aórtica e a musculatura ventricular mediante um enxerto anterior, que é suturado ao enxerto posterior. Para realizar esta intervenção, é necessário evitar a perfuração septal, a lesão da artéria coronária septal, ou a extensão da incisão no septo em um ponto tão distante que dificulte a reconstrução com o enxerto anterior.

Um importante aumento no diâmetro do anel valvar aórtico foi alcançado pela operação, o que possibilitou o implante de próteses valvares de diâmetro superior a do anel aórtico nativo. Isto é verdadeiro tanto para pacientes com valva nativa (Gráfico 4) quanto para portadores de prótese aórtica (Gráfico 5). Considerando-se que o grupo de pacientes com valva nativa incluía pacientes mais jovens (idade média 11,5 anos) do que os com estenose de prótese (idade média de 27,2 anos), podemos inferir que o procedimento pode ser utilizado com sucesso igualmente em crianças e adultos, na presença da valva aórtica nativa ou de prótese previamente implantada.

Diferentes próteses aórticas foram empregadas na presente série cirúrgica, obedecendo ao critério de opção do paciente (quanto a uso de bioprótese ou prótese mecânica) e de disponibilidade (relativa a diferentes modelos de prótese mecânica). Biopróteses e próteses metálicas que não apresentem estrutura com protusão inferior nos parecem adequadas para emprego na operação, pois oferecem reduzido risco de contato com enxertos de ampliação situados abaixo do plano valvar ou com a via de saída do ventrículo esquerdo. Não consideramos implante de homoenxerto nestes pacientes; acreditamos que isto seja possível, desde que os enxertos de ampliação sejam colocados de modo a resultar em estrutura tubular na região dos seios de Valsalva e aorta ascendente; com isto a aposição de folhetos aórticos deverá ficar adequada após sutura de comissuras de sustentação do homoenxerto.

A técnica de ampliação simétrica parece se enquadrar dentro dos requisitos fundamentais de um procedimento que vise a ampliação da via de saída do ventrículo esquerdo, quais sejam (31):

1) Não danificar o tecido de condução, o sistema coronário ou a valva mitral.

2) Apresentar mortalidade operatória aceitável.

3) Coadunar-se com o crescimento do paciente, mediante a possibilidade de se implantar uma prótese de tamanho grande ou permitir o crescimento do anel valvar.

4) Determinar uma diminuição consistente e marcada no gradiente entre o ventrículo esquerdo e a aorta, sem resultar em fenômenos embólicos ou disfunção ventricular progressiva.

5) Possibilitar reoperações futuras.

Ressalve-se que a ampliação posterior, conforme realizada em pacientes com valva mitral nativa, aborda o anel e o folheto anterior da valva; uma execução inadequada da técnica descrita poderá eventualmente determinar compromisso funcional da valva mitral, ao determinar encurtamento, deformação ou espessamento do folheto abordado.

Outro ponto é representado pelo risco de comprometimento da circulação coronária, conforme observado em um paciente necessitou revascularização miocárdica devido à obstrução de óstios coronários, complicação atribuível à reduzida dimensão de aorta íntegra proximal a origem das artérias coronárias, o que resultou da remoção da prótese existente. Uma reconstituição anelar com enxerto de pericárdio bovino que permitisse aumento da distância entre posição de fixação da nova prótese e óstios coronarianos seria o procedimento indicando para este paciente, conjuntamente com a ampliação de diâmetro do anel. Esta reconstrução foi realizada em pacientes desta série, devido a endocardite da valva removida ou a dano do anel aórtico durante remoção da prótese.

Referente à morbidade e mortalidade hospitalar (incluindo-se o período per-operatório), o resultado obtido parece satisfatório, especialmente se considerarmos a elevada prevalência de pacientes 
Sant'Anna J R, Kalil R A K, Prates P R, Jungblut C, Nesralla I A - Dupla ampliação anterior e posterior do anel aórtico para substituição valvar. Rev Bras Cir Cardiovasc 2002; 17 (1): 35-46.

com operação cardíaca prévia (73,1 \%). Além da complicação descrita, relativa ao compromisso de óstios coronários, registramos apenas uma reoperação por sangramento cirúrgico (não relacionado diretamente a técnica) e um óbito devido a choque séptico, ambos eventos em pacientes previamente submetidos a operação cardíaca com circulação extracorpórea. A mortalidade hospitalar global foi de $3,8 \%$, que compara favoravelmente com a observada na literatura para as diferentes técnicas de ampliação anelar aórtica.

Os resultados tardios, observados em período pós-operatório de 2 a 60 meses, são favoráveis. Para o período observado, nenhum paciente necessitou reoperação e a configuração da via de saída do ventrículo esquerdo e da aorta ascendente permanecem uniformes. Como nenhum paciente foi reoperado no período de avaliação, não podemos definir se a técnica poderá dificultar uma operação futura; nossa experiência prévia com reoperações em pacientes com implante de enxertos na aorta ascendente (tanto para ampliações anelares como em operações de tubos valvados) não parece indicar dificuldade adicional durante reoperação.
Pode ser questionado o número relativamente elevado de pacientes submetidos à operação descrita no período estudado, que foi de 6 anos. Contudo, foi efetuado em uma Instituição aonde são realizados todos os tipos de operações cardíacas, em número superior a 1500/ano. Além da clara indicação em crianças, visando reduzir o risco de uma desproporção futura entre dimensão corporal/tamanho da prótese, os resultados iniciais favoráveis encorajaram o emprego da técnica em pacientes com disfunção de prótese aórtica de causas diversas ou com gradiente transprotético elevado.

O procedimento da dupla ampliação anterior e posterior do anel aórtico não deve substituir técnicas bem conhecidas para este propósito, como a ampliação anelar posterior ou a aortoventriculoplastia; em realidade, situa-se em posição intermediária, sendo indicada quando se deseje um ganho de diâmetro do anel aórtico superior ao oferecido pela técnica de NIKCS et al. (1) ou MANOUGUIAN \& SEYBOLD-EPTING ${ }^{(2)}$, sem a necessidade de realizar a operação de Konno-Rastan ${ }^{(3,4)}$, um procedimento mais radical.

RBCCV 44205-570

Sant'Anna J R, Kalil R A K, Prates P R, Jungblut C, Nesralla I A - Anterior and posterior enlargement of the aortic annulus for valve replacement. Rev Bras Cir Cardiovasc 2002; 17 (1): 35-46.

ABSTRACT: Objectives: To present a technique for anterior and posterior enlargement of the aortic anulus using individual patches and results in 26 patients.

Method: Aortic valve surgery is performed with conventional cardiopulmonary bypass. For posterior enlargement, the longitudinal aortotomy is extended inferiorly and posteriosly in the mean portion of the noncoronary sinus, until the anterior mitral leaflet. Anterior enlargement results from incision between the two coronary ostium, extended in the left side of the ventricular septum for $2 \mathrm{~cm}$. Two patches of bovine pericardium are sutured in the distal portion of the anterior and posterior incisions and the aortic prosthesis is fixed. Aorta is reconstructed with the patchs. Combined cardiac defects are corrected.

Results: Procedure resulted in increase of the annulus diameter from $18.0 \pm 2.6 \mathrm{mmHg}$ to 24.5 $\pm 2.1 \mathrm{mmHg}(p<0.01)$. During operation, one patient required coronary revascularization owing to ostial obstruction. After the operation, one patient was reoperated owing bleeding (3.8\% morbidity) and one died with sepsys (3.8\% hospital mortality). In the late post-operative period (mean follow up: 24 months) there were no deaths or reoperations. Functional class improved for all followed up patients and 22 $(84.3 \%)$ are in class I (NYHA). For patients with stenosis of the native valve or prosthesis, mean peak systolic transvalvar gradient (measured by Doppler echocardiography) decreased from a preoperative value of $87.3 \pm 20.8 \mathrm{mmHg}$ to $25.9+10,3 \mathrm{mmHg}(\mathrm{p}<0.01)$.

Conclusion: Increase in diameter of the aortic annulus results from anterior and posterior enlargement using individual patches. The procedure should be considered for enlargement of the aortic annulus and reconstruction of the left ventricular outflow tract.

DESCRIPTORS: Aortic valve, surgery. Aortic valve stenosis, surgery. Heart valve prosthesis, implantation.

AGRADECIMENTOS: Este trabalho representa a continuidade da tese de Doutorado do Dr. João Ricardo M. Sant'Anna, apresentada ao Curso de PósGraduação do Instituto de Cardiologia do Rio Grande do Sul. Esta tese teve a orientação do Prof. Ivo A. Nesralla e como banca julgadora Profs. Noedir Stolf,
Milton Meyer e Renato A. K. Kalil. Seus comentários e sugestões contribuíram em muito no aprimoramento da experiência cirúrgica do autor e para redação do presente artigo. O Dr. Iran Castro realizou grande parte das avaliações ecocardiográficas que ajudaram a compor o resultado deste trabalho. 
Sant'Anna J R, Kalil R A K, Prates P R, Jungblut C, Nesralla I A - Dupla ampliação anterior e posterior do anel aórtico para substituição valvar. Rev Bras Cir Cardiovasc 2002; 17 (1): 35-46.

\section{REFERÊNCIAS BIBLIOGRÁFICAS}

1 Nicks R, Cartermill T, Berstein L - Hypoplasia of the aortic root: the problem of aortic valve replacement. Thorax 1970; 25: 339-46.

2 Manouguian S \& Seybold-Epting W - Patch enlargement of the aortic valve ring by extending the aortic incision into the anterior leaflet: new operative technique. $J$ Thorac Cardiovasc Surg 1979; 78: 402-12.

3 Konno S, Imai Y, lida Y, Nakajima M, Tatsuno K - A new method for prosthetic valve replacement in congenital aortic stenosis associated with hypoplasia of the aortic valve ring. J Thorac Cardiovasc Surg 1975; 70: 909-17.

4 Rastan H \& Koncz J - Aortoventriculoplasty: a new method for the treatment of left ventricular outflow tract obstruction. J Thorac Cardiovasc Surg 1976; 71: 920-27.

5 Lebabibi Z, Wu J R, Walls J T - Percutaneous balloon aortic valvuloplasty: results in 23 patients. $A m \mathrm{~J}$ Cardiol 1984; 53: 194-7.

6 Trusler G A, Moes C A, Kidd B S - Repair of ventricular septal defect with aortic insufficiency. J Thorac Cardiovasc Surg 1973; 66: 394-403.

7 Pereira E M, Costa A R, Santos M F, Daudt N S, Lara R F A - Avaliação pré-operatória do paciente candidato à cirurgia cardíaca. In: Nesralla I A, ed: Cardiopatias cirúrgicas para o ano 2000. São Paulo. Fundo Editorial Byk-Procienx. 1994; 229-43.

8 Lucchese F A, ed - Tratamento cirúrgico pós-operatório. São Paulo. Fundo Editorial Byk-Procienx, 1985; 474p.

9 Santanna J R - A pesquisa em cirurgia . Rev AMRIGS 1994; 38: 177-86.

10 Foster A H, Tracy C M, Greemberg G J, McIntosh C L, Clark R E - Valve replacement in narrow aortic roots: serial hemodynamics and long-term clinical outcome. Ann Thorac Surg 1986; 42: 506-16.

11 De Paulis R, Sommariva L, Russo F et al. - Doppler ecchocardiograpy evaluation of the CarboMedics valve in patients with small aortic annulus and valve prosthesis-body surface mismatch. J Thorac Cardiovasc Surg 1994; 81: 57-62.

Girardet R E \& Wheat M W Jr - Technique of aortic valve replacement. J Thorac Cardiovasc Surg 1976; 71: 446-9.

David T \& Uden D E - Aortic valve replacement in adult patients with small aortic annulli. Ann Thorac Surg 1983; 36: 577-83.

14 Antunes $\mathrm{M} \mathrm{J}$, Colsen P R, Kinsley R H - Intermittent aortic regurgitation following aortic valve replacement with the Hall-Kaster prosthesis. J Thorac Cardiovasc Surg 1982; 84: 751-4.

Birks $\mathrm{W}$ - Techniques in aortic valve implantation. In: Rábabo G \& Cooley D, ed. Heart valve replacement and future trends in cardiac surgery. New York: Futura Publishing, Inc, 1987; 69-81.

16 Blank R H, Pupello D F, Bessone L N, Harrison E E, Sbar $S$ - Method of managing the small aortic annulus during valve replacement. Ann Thorac Surg 1976; 22: 356-61.

17 Rittenhouse E A, Sauvage L R, Stamm S J, Mansfield P B, Hall D G, Herndon P S - Radical enlargement of the aortic root and outflow tract to allow valve replacement. Ann Thorac Surg 1979; 27: 367-73.
18 Tokunaka K, Togo T, Hamoda Y, Sadahiro M - Aortic annular enlargement to allow single or double valve replacement. Rinsho-Kybocu-Gaka 1984; 4: 463-72.

19 Osinowo O, Ross J K, Monro J L - Aortic root enlargement with glycerol preserved homologous dura mater patch during aortic valve replacement. Thorac Cardiovasc Surg 1985; 33: 23-5.

20 Meyer M, Lucchese F A, Coutinho J H et al. - Tratamento do anel aórtico pequeno: manuseio da desproporção anel aórtico/prótese. In.: $20^{\circ}$. Congresso Nacional de Cirurgia Cardíaca. Maceió, 1993. (Resumos).

21 Yener A, Ozdemir A, Sinav A, Yener N, Hauf E - A new technique to enlarge the aortic annulus. Ann Thorac Surg 1993; 55:1260-1.

22 Björnstad P G, Rastan H, Keutel J, Beuren A J, Koncz $\mathrm{J}$ - Aortoventriculoplasty for tunnel subaortic stenosis and other obstructions of the left ventricular outflow tract. Circulation 1979; 60: 59-69.

23 Flemming W H \& Sarafian L B - Aortic valve replacement with concomitant aortoventriculoplasty in children and young adults: long-term follow-up. Ann Thorac Surg 1986; 42: 506-16.

24 Frommelt $\mathrm{P}$ C, Lupinetti $F$ M, Bove $E$ L Aortoventriculoplasty in infants and children. Circulation 1992; 86(Suppl): II-176-80.

25 McIntyre B, Guyton R A, Jones E L, Crave J M, Williams W H, Hatcher C R Jr - Reoperation for prosthetic valve degeneration after Konno aortoventriculoplasty. $J$ Thorac Cardiovasc Surg 1986; 91: 934-36.

26 Yamaguchi $\mathrm{M}$, Ohashi $\mathrm{H}$, Imai $\mathrm{M}$, Oshima $\mathrm{Y}$, Horokawa $Y$ - Bilateral enlargement of the aortic ring for valve replacement in children: new operative technique. $J$ Thorac Cardiovasc Surg 1991; 102: 202-6.

27 Clark D R - Extended aortic replacement for treatment of left ventricular outflow tract obstruction. $J$ Cardiac Surg 1987; 2: 121-8.

28 Ross D N - Pulmonary valve autotransplantation (The Ross operation). J Thorac Cardiovasc Surg 1988; 3(Suppl): I-313-9.

29 Gerosa G, McKay R, Ross D N - Replacement of the aortic valve or root with a pulmonary autograft in children. J Thorac Cardiovasc Surg 1991; 51: 424-9.

30 Mckowen R L, Campbell D N, Woelfel G F, Wiggins J W Jr, Clarke D R - Extended aortic root replacement with aortic allografts. J Thorac Cardiovasc Surg 1987; 93: 366-74.

31 Demmy T L \& Magovern G J - Aortic root enlargement procedures. In: Emery RW \& Arom KV, ed. The aortic valve. Philadelphia: Hanley \& Belfus, Inc. 1991. p.253-61.

32 Katsumata $\mathrm{T}$, Kurosawa $\mathrm{H}$, Koyanagi $\mathrm{H}$ - Intra-arterial aortoinfundibuloplasty: hemodynamic and anatomical study of a new method for the enlargement of a small aortic annullus. J Cardiac Surg 1993; 8:125-9.

33 Doty D B, Polansky D B, Jenson C B - Supravalvular aortic stenosis: repair by extended aortoplasty. $J$ Thorac Cardiovasc Surg 1977; 74: 362-71.

34 David T E, Komeda M, Brofman P R - Surgical treatment of aortic root abcess. Circulation 1983; 80 (Suppl): I-1269-74. 